\title{
32
}

\section{A Measurement Software for Vision Machine Applications}

\author{
L. C. R. Carpinetti
}

Escola de Engenharia de São Carlos -USP

C.P. 359, 13560-970 São Carlos SP Brazil, FAX: +55 16271 9241, email: carpinet@tigre-prod.prod.eesc.sc.usp.br

R. Spragg

Cranfield University - Bedford, MK 43 OAL, England, FAX: +44 234 750875

\begin{abstract}
This paper presents the development of a software for measurement of linear and angular dimensions and geometric deviations of two-dimensional views of prismatic and cylindrical components. This software was developed in cooperation with Integral Vision, a Software House based in Bedford, England, for application in vision systems. The software organization and its different modules are presented. A final discussion is made in which the merits and drawbacks of such a measurement system is considered.
\end{abstract}

\section{Keywords}

Machine Vision, Metrology Software 


\section{INTRODUCTION}

Machine Vision systems are playing an increasingly important role in automated inspection. This technology is being used for such diverse applications as looking for defects in parts, ensuring correct configuration of parts in assembly lines and dimensional inspection. They bring special advantages to the automated factory such as high speed of measuring and inspection, high flexibility, in process, on-line inspection and low cost. In addition to this, the contactless type of inspection and the ability to inspect small or restricted areas make such a measurement system appropriate in many inspection situations.

Applications of machine vision in automated inspection cells are well demonstrated in the automotive industry. A number of automotive plants, including General Motors of Canada, Chrysler Motors Newark (DE) (Pastorius, 1989) and Austin Rover (UK) (Mullins, 1987) have implemented vision systems for 100 percent dimensional process control of car body assembly. These systems provide measurement capabilities not previously available to the car body assembly plants.

At Austin Rover (UK), for example, a vision inspection cell inspects car body dimensions to an accuracy of $0.1 \mathrm{~mm}$. Operating on a two minute cycle time, 62 laser cameras make 88 checks on the body shell (Mullins, 1987).Moreover, recent developments in the field of image analysis are increasing the resolution and accuracy of vision systems.

In the field of Image and Vision Computing, the approximation of digitized curves into segments and arcs is an image analysis technique presently available (Rosin e West, 1989). Roche (1989) offers new evidence of applying this technique for automatic segmentation of digitized profiles of objects. This article reports the development and implementation of a vision machine measurement software (Carpinetti, 1990) that further refine the results of a procedure for automatic segmentation of a contour of an object, (Roche, 1989), defined by a set of points extracted by using a video camera and a vision system for edge detection.

\section{MACHINE VISION SYSTEMS IN INSPECTION AND DIMENSIONAL MEASUREMENT}

Machine Vision systems basically employ digital representation of the scene under investigation (Nash and Webster, 1984). In a typical arrangement, the camera viewing area is divided into a matrix of picture elements called pixel. The larger is the number of pixels the higher is the resolution. Pixels are typically structured in arrays of 256.000 (512 x 512 matrix); the array size depends upon the hardware utilized.

Once the image of a part is spatially sampled by each pixel, its signal is sent to the processor and treated by image analysis techniques implemented in software. Digital data produced by vision systems may be either binary or gray scale. Binary systems translate each pixel into 
either black or white. Gray scale systems use a certain number of bytes of information for each pixel to record different levels of light intensity. Because of the amount of information to be processed, gray scale systems are slower than binary systems and require more memory to manipulate the data.

The cameras used are basically of two types: Vidicon and solid state cameras. Vidicons are similar to closed circuit television cameras. They produce an analog signal that must be converted to the digital format. Solid state charge couple device (CCD) and charge injection device (CID) cameras are based on integrated circuit technology and provide digital information with no need for translation. As a result, they provide more accurate information than a Vidicon camera does because of the distortion that analog to digital conversion produces. By changing the focus of the camera, or by using different arrangements of lenses, users can get higher resolution over a smaller area, or can look at a larger area with less resolution.

Vision systems generally rely on one or two forms of illumination: backlighting or frontlighting. Backlighting produces a high contrast image of the part's silhouette. An advantage of backlighting is speed: it produces less information than other sources of illumination and so requires less time for analysis. Binary systems usually use backlighting illumination because of the need for high contrast. But backlighting is impractical in many industrial situations or in cases requiring inspection of features that do not have silhouettes. Frontlighting depends on reflected light from the part being inspected. Unlike backlighting, frontlighting can provide information about the surface of the part. Features to be inspected must be distinguished by enough light intensity to neutralize any light variation coming from the environment. Common sources of light include incandescent and fluorescent lamps, lasers, strobe lamps, radiant energy from a heated part and arc lamps.

The operations performed by a machine vision system in digitizing the profile of an object for posterior measurement may be divided as follows:

- image acquisition;

- image binarization;

- extraction of the pixel coordinates of the profile;

- conversion of pixel coordinates into real world coordinates.

The accuracy of machine vision systems is dependent on the resolution at which the image of the object is digitized and the algorithms for extraction of the pixel coordinates of the profile.

\section{IMPLEMENTATION OF SOFTWARE FOR METROLOGY}

For any particular measurement, the software implementation comprises the following steps: 
1. mathematical modelling: Mathematical modelling, for metrology, may be defined as the construction of a system of mathematical relationships or equations that connect the measurements made in the test procedure to the various assessment parameters which describe the geometric properties of the workpiece. For example, the British Standard Institution (BSI, 1989) recommends that a line in a specified plane should be defined by either one point on the line and information about the orientation of the line or by two points on the line. Another example, the departure of any point from a straight line is given in terms of the "euclidian" distance between the point and the line. The mathematical modelling should have the property that small changes in the geometric element result in correspondingly small changes in the equation parameter values.

2. numerical analysis: numerical calculations are employed to find the position and orientation of the geometric element that best fits the measured points. The fit is then represented by the value of the parameters of its mathematical model. For example, a set of points taken around one section of a cylinder will define the centre coordinates and the radius of a circle, which are the parameters of its equation. Erroneous computation of these parameters will result in poor estimation of the geometric references and will strongly affect the software accuracy [Cox, 1985]. Different criteria for specifying the best fit are possible. In general, the criterion should be to make some combination of the residuals (or departure of each point from the reference) as small as possible. The British Standard Institution (BSI, 1989) and the National Physical Laboratory (Forbes, 1989) recommend the least-squares technique for computing the best fit geometric element to data. Establishing a least-square best-fit geometric element to the data is based on the minimization of the summation of the square of departures of each point from reference, hence the term least-squares fit. This technique is quite used and extensive literature regarding it is available.

3. design of algorithm: Any particular software for metrology will entail several separate tasks: eg. collecting data, checking data, setting up equations, computing the assessment parameters and their statistical uncertainties. It is very desirable that the software be wellstructured, or modular, in the sense that such natural divisions be reflected in it by keeping each in a separate module (subroutine). Such modules, where appropriate, can themselves be subdivided into smaller modules (Anthony and Cox, 1984].

\section{MEASUREMENT SOFTWARE SPECIFICATIONS}

The Measurement Software was specified for two dimensional applications only. This is quite adequate for many industrial applications, since many situations involve a two dimensional view. The input is a set of measured points previously obtained by a machine vision. The output is the result of the assessment. The process is schematically shown in figure 1.

The procedure for the automatic segmentation of profiles was incorporated into the measurement system as it was originally proposed (Roche, 1989). This procedure calculates a 
first approximation of the original set of points into geometric entities, each entity being either an arc or a segment. The steps for this procedure are as follows:

- Open the file containing the set of measured points.

- Proceed the vectorisation and segmentation.

- Save the results in a text file.

These operations are carried out for one contour at a time. If, however, an object having many contours is to be dealt with, these operations may be applied for each contour and then combined into the same file. The procedure to obtain a segmented contour is based on approximation, which depends on the parameter-of-vectorisation (for reducing the original curve in a contour of vectors) and the parameter-of-segmentation (for approximating the contour of vectors for extraction of boundaries and curvature calculations). The right choice of parameters is a key requirement in achieving the correct representation of the contour in terms of arcs and lines.

Figure 1 Overview of the process.

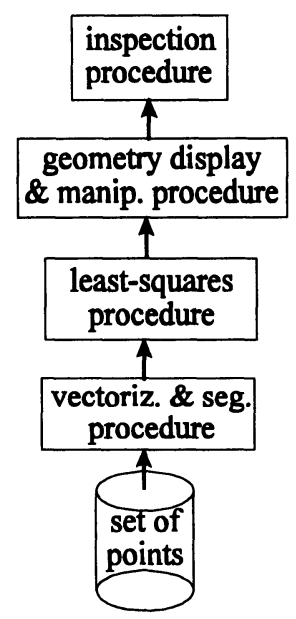

In order to investigate dimensions and geometric shape of a profile, this approximation method can be further refined by applying a curve fitting procedure which can allow a more accurate approximation of the centre and radius of arcs as well as the slope of lines. Therefore a least squares curve fitting procedure is proposed. The least-squares fitting is proceeded by following the steps bellow:

- Read the file containing the set of measured points.

- Read the file containing the results of the vectorisation and segmentation process. 
- Proceed the least-squares fitting by taking in to account the set of original points between the two boundaries of each entity.

- Keep the results in a array of geometric elements for posterior use or save in a file.

After this, the user selects the features to be inspected. The functions for inspection are presented to the user through the User Interface in a menu selection mode.

The set of points describing any profile of a workpiece is assumed to be contained in a binary file. The points are defined in terms of cartesian coordinates. This file is used as input to the vectorisation and segmentation procedure, as well as to perform the Least-Squares Fitting procedure.

The Software structure is schematically shown in figure 2. Internally, the software is basically organized in three different layers, which correspond to different levels of action as well as different functions.

\section{USER INTERFACE}

METROLOGY LAYER

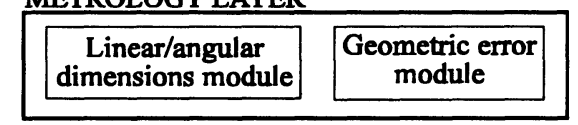

GEOMETRY LAYER

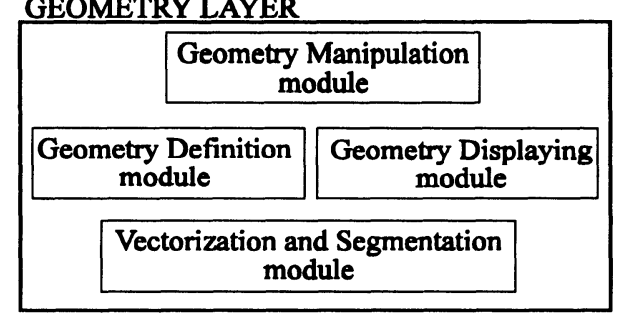

Figure 2 Software organization.

The Geometry Layer is the bottom level of the software, concerned with the definition, manipulation and displaying of the geometric elements used as references at the metrology level.

The Geometry layer is divided in four modules:

1. Vectorisation \& Segmentation Module: containing the procedure for automatic segmentation of profiles as proposed by Roche (1989). The results of the Vectorisation and Segmentation procedure are stored in an array of geometric elements. The information for 
each geometry entity includes its type (either a line or an arc) and the indexes of the extremity points in the array of $(x, y)$ coordinates.

2. Geometry Definition Module: encompassing the mathematical functions for:

- computing the Least-Squares best fit geometric elements to cartesian data points. The algorithms adopted employ stable parametrization of geometric elements and are based in numerical algorithms proposed by Forbes (1989). The specific geometries considered in this work are lines, circles and arcs in a specific plane. The information about the extremities points of each geometric entity returned by the vectorisation and segmentation procedure is used to select the points pertaining to each entity in the array of data points, and the entity type is used in applying the apropriate least squares algorithm (either line or arc). In the case that the entity type is an arc and the first and last points coincide, then a least squares circle algorithm is applyed.

- redefine the intersection points between the entities redefined by the least squares procedure.

3. Geometry Manipulation Module: containing the functions for manipulation of the geometric entities, such as opening and closing the file of geometric entities, selecting, retrieving and putting them back into the file.

4. Geometry Display Module: containing the hardware dependent functions for displaying of the geometric references.

The Metrology Layer is the middle level layer, concerned with the evaluation of position, size and deviation from nominal dimensions of the geometric elements previously defined in the geometry layer. It is in turn divided in two modules:

1. functions for measurement of linear/angular dimensions: the mathematical modelling of these functions are based on planar analytic geometry. The basic functions involved in performing measurement of distances/angles are for:

- measurement of distance from point to line: the normal distance from a point to a line. The point may be the centre of a circle, the peak of an arc, or the intersection between any two geometries.

- measurement of distance from point to point: the distance may be the $\mathrm{x}$ projection, the y projection or the actual distance. The points may be the centre of a circle, the peak of an arc or the intersection between two geometries

- measurement of distance from line to segment: by taking the average of the normal distance of the extremity points from the line.

- measurement of distance between two segments: by taking the average of the normal distance of the extremity points of each segment from the opposite one;

- measurement of angles: the angle between two lines. 
2. functions for evaluation of geometric errors: the mathematical modelling for the calculation of the errors are based on definitions of geometric errors stated in national standards (BSI, 1990, BSI, 1989) and on planar analytic geometry. The inspection of geometric errors is divided in:

- measurement of form error: straigthness and roundness;

- measurement of attitude error: parallelism, squareness and angularity;

- measurement of location error: concentricity and symmetry;

The User Interface is the top level layer, through which the users and computer interact. The User Interface adopted is of a menu selection type. Menu selection is regarded as a mode of operation well suited to human-computer interaction, as it can eliminate training and memorization of complex command sequences (Sutchliffe, 1988). The menu structure is organized in layers, converging on specific actions. The first layer is concerned with the presentation of the possible types of measurement and a list of options of features which may be inspected (figure 3). The terminology used is based on definitions given in international standards (BSI, 1990). The second layer is mainly concerned with data acquisition.

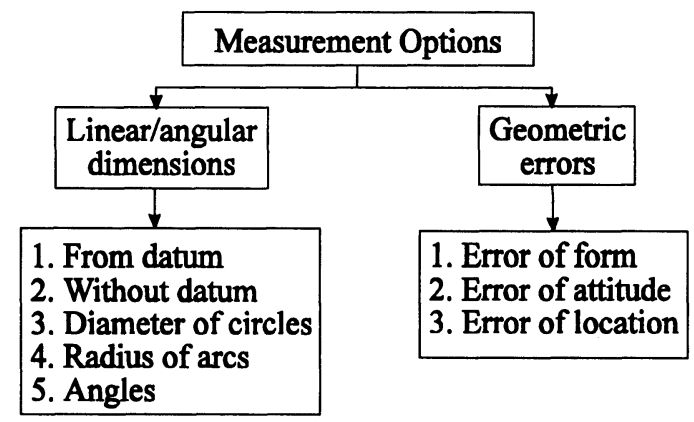

Figure 3 Measurement options.

\section{MEASUREMENT SOFTWARE IMPLEMENTATION AND TESTS}

C language was adopted for implementing this software,since it is being used more and more in industry, as it provides several advantages, viz: powerful structural features; standard dynamic memory management; low and high level functions and modularity. 
The software was implemented in cooperation with Integral Vision Ltd, Bedford, England, a software-house in the field of vision technology. Therefore, some of its in-house facilities and product definition software tools were adopted for reasons of efficiency and existing software compatibility. These are as follows:

- The menu windows structure used for interfacing the system with the user was created by using the company's software libraries for windows set up and display (Integral Vision, 1990).

- The functions for graphic display were based on the company's low level software library (Integral Vision, 1989) and computer hardware facilities.

The computer used was an IBM PC AT compatible. A remote monitor was used due to the impossibility of working with the computer monitor in graphical mode (software limitation). The monitor was a HITACHI VM 900 monochrome, 500 lines, 23 centimetres. It was linked to the computer by a Visionetics VFG 512 grey level framestore of 512 by 512 pixels.

A file containing the set of measured points was generated by using a two-dimensional machine vision system (Auto-Vision ${ }^{\circledR}$, by Integral Vision Ltd). This system is based on a microcomputer IBM AT compatible, with a Visionetics VFG framestore, 512 by 512 pixels in 256 grey levels. The camera was a Pulnix monochrome CCD, 510 by 580 pixels.

The output of the image aquisition operation is a matrix of pixels, each pixel having a value varying from 0 to 255 defining the light intensity of the associated portion of the scene.

The light intensity of each pixel is reduced to either black or white (image binarization), depending on whether the light intensity exceeds a given threshold level.

The extraction of the pixel coordinates of the profile is achieved by using an edge detection algorithm called "sniff". A line is defined crossing the contour. This line is then scanned, by using the sniff algorithm, until a transition from a white pixel to a black pixel or vice versa is founded. This transition is followed all around the shape of the object and the position of every pixel belonging to the transition is saved in dynamic memory.

The conversion of pixel coordinates into real world coordinates is accomplished by multiplying the $\mathrm{x}$ and $\mathrm{y}$ pixel coordinates by a transformation matrix relating the size of one picture element to the size of the object contained in a picture element.

After this, the set of data points describing a particular profile is available for measurement. The results of the measurement procedures are saved in a file for posterior analysis. The results are saved in sequence, following the sequence that the measurements are performed. The information saved, for each measurement performed is as follows:

- for linear/angular dimensions: nominal dimension, lower and upper tolerances and actual dimension;

- for geometric shape: geometric tolerance and actual geometric error 
Tests for correctness were carried out testing each function inside a module separately and then testing it in its particular context. The functions for measurement of linear/angular dimensions and geometric errors were tested for correctness and the results were verified to be satisfactory, although the results were affected by the accuracy of the machine vision in the edge detection and calibration processes.

\section{DISCUSSION AND CONCLUSION}

The low level functions used for setting up and displaying the menus imposed setting the computer monitor in text mode and the use of a remote monitor for graphic display. This led to a less standard hardware configuration. This disadvantage can be solved by using more sophisticated user interface software, running in graphical mode and with pop-up/pull-down menus.

This measurement system was implemented in manual mode. In other words, the user has to go through the menus each time a measurement is to be performed. However, this can be automated for example by implementing a function chart for sequential processes. In this case, the user goes through the menus only during the set up phase, and then the defined sequence of options are saved for posterior automatic execution.

The execution time for the automatic segmentation, least squares curve fitting and measuring functions were of the order of tenths of a second in the worst cases. The overall execution time was not possible to measure due the fact that the set of measured points taken by the machine vision were first saved in a file for subsequent use. However, this step can be avoided by interfacing the machine vision with the measurement system and keeping the results in dynamic memory.

Although the accuracy of measurement of machine vision systems is unsatisfactory for high precision measurements, it is very much adequate in cases requiring low to medium precision, therefore making vision technology an alternative in many dimensional inspection situations.

The inclusion of the automatic segmentation technique in the measurement software does improve the apropriateness of vision systems in automated inspection activities.

The implementation of the procedure for application of the Least-Squares Curve Fitting Method on the results of the automatic segmentation procedure resulted in a better approximation of the geometric elements and therefore improved the accuracy of the measurement system as a whole.

The limitation of this Software to measurement of prismatic and cylindrical workpieces does not invalidate the usefulness of the system as these encompass the majority of the sort of shapes of manufactured components.

Finally, the Measurement Software, as it was implemented is machine independent, in the sense that several "measuring machines" can be used in the data acquisition task and integrated under the same measurement software. 


\section{REFERENCES}

Anthony, G. T. and Cox, M. G. (984) The design and validation of software for metrology, National Physical Laboratory, Londres, NPL Report DITC 50/84.

BSI (1990) Engineering drawing practice. Part 3: Recommendations for geometric tolerances, London: British Standard Institution. BS 308: Part 3.

BSI (1989) British standard guide to assessment of position, size and departure from nominal form of geometrical features, British Standard Institution, Londres, BS 7172.

Carpinetti, L. C. R. (1990) Development of a Machine Independent Measurement Software. Msc thesis, Cranfield Institute of Technology.

Cox, M. G. (1985) Software for computer-aided measurement. Laboratory Practice, June, 59 $-63$.

Forbes, A. B.(1989) Least squares best fit geometric elements. National Physical Laboratory, Londres, NPL Report DITC 140/89.

Integral Vision (1989) Auto Vision library, (program). Beta release. Bedford, UK: Integral Vision,Ltd.

Integral Vision (1990) Miscell library, (program). Beta release. Bedford, UK: Integral Vision,Ltd,1990.

Mullins, P.(1987) European quality in production Production, 99(12), 50 - 53.

Nash, W. L. and Webster, F. M. (1984) Machine vision systems today and tomorow. Quality progress, 17 (6), 13 - 17.

Pastorius, W. J.(1989) Vision measures up in automated assembly. Manufacturing Engineering,April, 81 - 83.

Roche, H. (1989) Automatic segmentation of profiles resulting in a perfect geometrically defined, continuous and bounded object. Msc thesis, Cranfield Institute of Technology.

Rosin, P. L. and West, G.(1989) Segmentation of edges into lines and arcs. Image and Vision Computing, 7 (2), 109 - 114.

Sutcliffe, A.(1988) Human-computer interface design. Macmillan Ed. Ltd, 156 - 180.

\section{BIOGRAPHY}

Dr. L. C. R. Carpinetti is an assistant professor of quality control at the University of São Paulo where he teaches statistical quality design and process control. He received a M. Sc. in metrology and quality assurance from University of Cranfield, $\mathrm{UK}$, and a $\mathrm{PhD}$ in engineering from University of Warwick, UK. 
Prof. R. Spragg has recently retired as professor of metrology and quality assurance at the University of Cranfield, UK. He also worked for several years as director of Rank Taylor Hobson Limited, a British manufacturer of precision systems. 